\title{
Posttranscriptional control of PLOD1 in adipose-derived stem cells regulates scar formation through altering macrophage polarization
}

\author{
Miao Xu ${ }^{1}$, Shuo Fang ${ }^{2}$, Aiguo $\mathrm{Xie}^{3}$ \\ ${ }^{1}$ Department of Plastic and Reconstructive Surgery, Xinhua Hospital, School of Medicine, Shanghai Jiao Tong University, Shanghai, China; \\ ${ }^{2}$ Department of Plastic and Reconstructive Surgery, Shanghai Changhai Hospital, Naval Medical University, Shanghai, China; ${ }^{3}$ Department of \\ Plastic and Reconstructive Surgery, Shanghai Ninth People's Hospital, School of Medicine, Shanghai Jiao Tong University, Shanghai, China \\ Contributions: (I) Conception and design: M Xu; (II) Administrative support: M Xu, A Xie; (III) Provision of study materials or patients: M Xu, A Xie; \\ (IV) Collection and assembly of data: All authors; (V) Data analysis and interpretation: All authors; (VI) Manuscript writing: All authors; (VII) Final \\ approval of manuscript: All authors. \\ Correspondence to: Aiguo Xie. Department of Plastic and Reconstructive Surgery, Shanghai Ninth People's Hospital, School of Medicine, Shanghai \\ Jiao Tong University, 639 Zhizaoju Road, Shanghai 200011, China. Email: nrxdf58777@yeah.net.
}

Background: The level of cutaneous scar formation is a critical parameter to determine the success of skin
wound healing. Adipose-derived mesenchymal stem cells (AMSCs) have been applied to improve treatment
of cutaneous injury with the purpose of reducing scar formation.
Methods: The levels of procollagen-lysine 1,2-oxoglutarate 5-dioxygenase 1 (PLOD1) were assessed at scar
sites. Then, PLOD1 in AMSCs was depleted by either expression of a PLOD1-specific short-hair interfering
RNA (shPLOD1) or by expression of microRNA-449 (miR-449) that targets and suppresses protein
translation of PLOD1 through 3 prime untranslated region (3'-UTR) interfering. For induction of skin
injury, a blade cut of 1.5 -cm long and 2-mm thick was made on the middle back of the mice. Transplantation
of either AMSCs-shPLOD1 or AMSCs-miR-449 into the injured region of the mice was performed via tail
vein injection. The fibrosis as well as underlying mechanisms were assessed.
Results: The AMSCs expressed high levels of PLOD1, a potent stimulator of fibrosis. We knocked down
PLOD1 in AMSCs by expression of either shPLOD1 or miR-449. Transplantation of either AMSCs-
shPLOD1 or AMSCs-miR-449 significantly reduced the fibrotic process in the injured region of the mice to
a similar degree. Mechanistically, transplantation of either AMSCs-shPLOD1 or AMSCs-miR-449 shifted
macrophage polarization from M2 to M1-like and reduced both reactive oxygen species (ROS) and activation
of myofibroblasts from fibroblasts.

Conclusions: Suppression of PLOD1 levels in AMSCs either directly by shPLOD1 or indirectly by miR449 may substantially improve the anti-fibrotic potential of AMSCs during wound healing, likely through altering macrophage polarization.

Keywords: Scar formation; adipose-derived mesenchymal stem cells (AMSCs); macrophages; procollagen-lysine 1,2-oxoglutarate 5-dioxygenase 1 (PLOD1); miR-449

Submitted Aug 31, 2021. Accepted for publication Oct 16, 2021.

doi: $10.21037 / \mathrm{atm}-21-4978$

View this article at: https://dx.doi.org/10.21037/atm-21-4978 


\section{Introduction}

The consequence of tissue regeneration and repair in many circumstances appears to be the replacement of the damaged cutaneous tissue with a pathological connective tissue, defined as a scar (1). During skin wound healing, deposition of the extracellular matrix (ECM) occurs in the parenchymal tissue, and excess ECM may lead to scar formation (1). As some necessary dermis structures are missing from skin scar tissue, it does not function like the surrounding skin (2). Moreover, the skin tensile strength of the skin scar tissue is also significantly reduced, resulting in a susceptibility to re-injury (3).

Scars are characterized by collagen fiber rearrangement, activation of $\alpha$-smooth muscle actin ( $\alpha$-SMA)-expressing myofibroblasts, and high levels of transforming growth factor $\beta 1$ (TGF- $\beta 1)$ that is produced and secreted in the affected tissue (4). Procollagen-lysine 1,2-oxoglutarate 5 -dioxygenase 1 (PLOD1) plays a key role in the regulation of lysine residue hydroxylation in collagen telopeptides as well as in the cross-link formation of collagen pyridinoline during fibrosis (5). Moreover, it is known that macrophages are essential during fibrosis and their M2-polarized forms are fibrotic (6).

Recently, cell-based therapy stemming from tissueengineering techniques has appeared an attractive method for improving patient wound healing (7). Among all types of cells to be used in preventing scar formation, mesenchymal stem cells (MSCs) have been most commonly studied and applied, attributable to their easy access, abundance, as well as their acceptable functioning during tissue repair and regeneration (8). Specifically, adipose-derived mesenchymal stem cells (AMSCs) are abundant, accessible, and have good characteristics for tissue repair (9-11). The AMSCs may function in normal wound healing through production and secretion of trophic factors to enhance regeneration and adapt inflammation in wound-associated fibrosis (12-15). Interestingly, recent studies have demonstrated that genetic modification of MSCs may further improve through their therapeutic effects.

MicroRNAs (miRNAs) are short non-coding RNAs that combine with the 3 prime untranslated region ( $3^{\prime}-$ UTR) of the target genes by nuclear acid pairing to silence genes due to altered spatial structure (16). Specifically, miR-449 is a miRNA that was found to regulate Notch signaling (17), cyclin-dependent kinase (CDK)/ retinoblastoma $(\mathrm{Rb}) / \mathrm{E} 2$ transcription factor $1(\mathrm{E} 2 \mathrm{~F} 1)(18)$, and RET kinase/Wnt signaling (19). On the other hand, although AMSCs and PLOD have been shown to play critical roles in wound healing $(20,21)$, the posttranscriptional control of PLOD1 by miR-449 in AMSCs as well as its role in regulation of fibrosis and scar formation has not been reported.

Here, we used genetic tools to enhance the therapeutic potential of AMSCs in preventing experimental scar formation. We present the following article in accordance with the ARRIVE reporting checklist (available at https:// dx.doi.org/10.21037/atm-21-4978).

\section{Methods}

\section{Protocols and animals}

The present study was approved by the Research and Animal Ethics Association of Shanghai Jiao Tong University (No. XHEC-F-2021-063), in accordance with the guidance from Shanghai Jiao Tong University for the Care and Use of Laboratory Animals, and a protocol was prepared before study without registration. Male and female 12 -weekold C57BL/6 mice (about 25 and 20 g, respectively) were purchased from Shanghai Laboratory Animals Center (SLAC) Laboratory Animal Co. Ltd. (Shanghai, China). Both male and female mice were used and distributed evenly in each experimental group to exclude a possibility of gender effects on the interpretation of the results. Mice were housed under a 12-h light-dark cycle. For the in vivo mouse experiment, power calculations $(\mathrm{P}<0.05)$ were performed to include exactly sufficient animals for the observed effects to be legitimate. An allocation concealment method was used, and efforts were made to ensure that potential confounders were minimized. No criteria were used for excluding animals (or experimental units) during the experiment, and no data were excluded during the analysis. The study did not have humane endpoints. For induction of skin injury, a blade cut of $1.5-\mathrm{cm}$ long and 2 -mm thick was made on the middle back of the mice. The mice were allocated to 3 groups: Group 1: mice received injury and transplantation of control AMSCs-scrambled sequence (AMSCs-scr); Group 2: mice received injury and transplantation of AMSCs-miR-449; and Group 3: mice received injury and transplantation of AMSCs-short-hairpin small interfering RNA for PLOD1 (shPLOD1). We have previously compared non-transfected AMSCs to AMSCsscr and did not find difference between these two cells in terms of the therapeutic potential here. Therefore, we did not include this control group additionally. 


\section{Preparation and differentiation of AMSCs}

Adipose tissue was obtained from the dorsocervical subcutaneous region of 12 -week-old C57BL/6 mice. The adipose tissue was carefully rinsed then incubated with $0.25 \%$ Trypsin (Sigma-Aldrich, St. Louis, MO, USA) in a $200-\mathrm{rpm}$ rotator at $37^{\circ} \mathrm{C}$ for $10 \mathrm{~min}$ to be digested. The digests that passed through a $100-\mathrm{mm}$ filter were resuspended in Dulbecco's modified Eagle medium (DMEM) supplemented with $15 \%$ fetal bovine serum (FBS). A positive selection for Stro- $1^{+}$cells (Becton, Dickinson, and Co. Biosciences, San Jose, CA, USA) by flow cytometry was done after 10 passages of the cells. A positive clone was then used for chondrogenic, osteogenic, and adipogenic differentiation assays for determining phenotype of the AMSCs, using specific differentiation toolkits [American Type Culture Collection (ATCC), Rockville, MD, USA; catalog number: PCS-500-052, PCS-500-050 and PCS500-051]. Mouse embryonic fibroblasts (MEFs) were purchased from ATCC (Catalog number: SCRC-1040). Human skin fibroblasts (HSFs) were purchased from ATCC (catalog number: SCRC-1041). Recombinant mouse TGF- $\beta 1$ (Sigma-Aldrich, USA) was added to cultured cells at a dose of $20 \mathrm{ng} / \mathrm{mL}$.

\section{Transfection and transduction of AMSCs}

Transfection of AMSCs were done using plasmids carrying miR-449, or antisense for miR-449 (as-miR-449), or a scr as a control, with Lipofectamine 3000 reagent (Invitrogen, St. Louis, MO, USA). The AMSCs were transduced with an adeno-associated virus (AAV) carrying shPLOD1 and a green fluorescent protein (GFP) reporter (connected with a $2 \mathrm{~A}$ sequence) under a cytomegalovirus (CMV) promoter, or an AAV carrying miR-449 and a GFP reporter (connected with a $2 \mathrm{~A}$ sequence) under a CMV promoter, or a control AAV carrying a GFP reporter and scr. Human embryonic kidney 293 cell line (HEK293, ATCC) was cotransfected with a pAAV-CMV-GFP plasmid (Clontech, Mountain View, CA, USA), a packaging serotype 2 plasmid (with rep and cap genes), and a adenovirus helper plasmid (Applied Viromics, LLC., Fremont, CA, USA) to produce the viruses. The AMSCs cells were incubated with AAVs at a multiplicity of infection (MOI) of 300 for $5 \mathrm{~h}$ to transduce the cells. At $24 \mathrm{~h}$ after infection, purification of the transduced $\mathrm{GFP}^{+}$cells were performed by flow cytometry.

\section{Flow cytometry}

For flow cytometric analysis, GFP was detected by direct fluorescence. Macrophages and M2 macrophages were detected by F4/80 and CD163, respectively. Data were analyzed using FlowJo software (FlowJo LLC., Ashland, OR, USA).

\section{Transplantation of AMSCs}

The transplantation of $2 \times 10^{6}$ shPLOD 1 -transduced AMSCs, or miR-449-transduced MSCs, or control AMSCs in a $150-\mu \mathrm{L}$ normal saline were injected into the tail vein of the mice 3 days before injury formation. This intravenous transplantation method required time for cell homing and was thus done 3 days before injury.

\section{Histology}

A Trichrome Stain (Masson) Kit (Sigma-Aldrich, USA) was used to perform Masson-trichrome staining on experimental tissue.

\section{Dihydroethidium (DHE) assays for reactive oxygen species (ROS) production}

A DHE assay was used to assess ROS production, and signals were detected and quantified at excitation/emission $520 / 610 \mathrm{~nm}$ by fluorometer (Thermo Fisher Scientific, Waltham, MA, USA).

\section{Enzyme-linked immunosorbent assay (ELISA)}

Proteins were isolated from cultured cells or mouse skin tissue at the injured site. Specific protein levels were detected by anti-PLOD1, anti-ROS, anti- $\alpha$-SMA, antiTGF- $\beta 1$, anti-collagen I ELISA kits (R\&D, Carpinteria, CA, USA).

\section{Quantitative polymerase chain reaction}

Total RNA extraction used a miRNeasy mini kit (Qiagen, Hilden, Germany) and quantitative reverse transcription polymerase chain reaction (RT-qPCR) used a QuantiTect SYBR Green PCR Kit (Qiagen). Primers were all purchased from Qiagen. Data were assessed using $2^{-\Delta \Delta \mathrm{Ct}}$ method. Relative levels of genes were obtained through sequential 


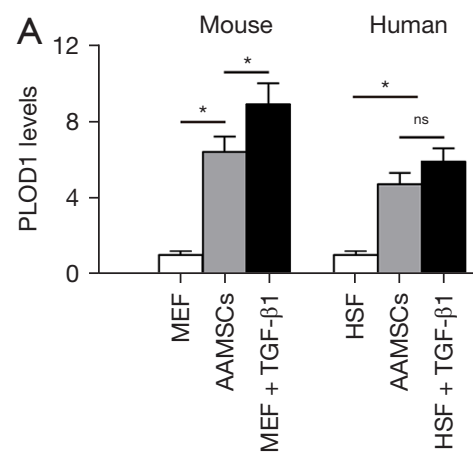

B
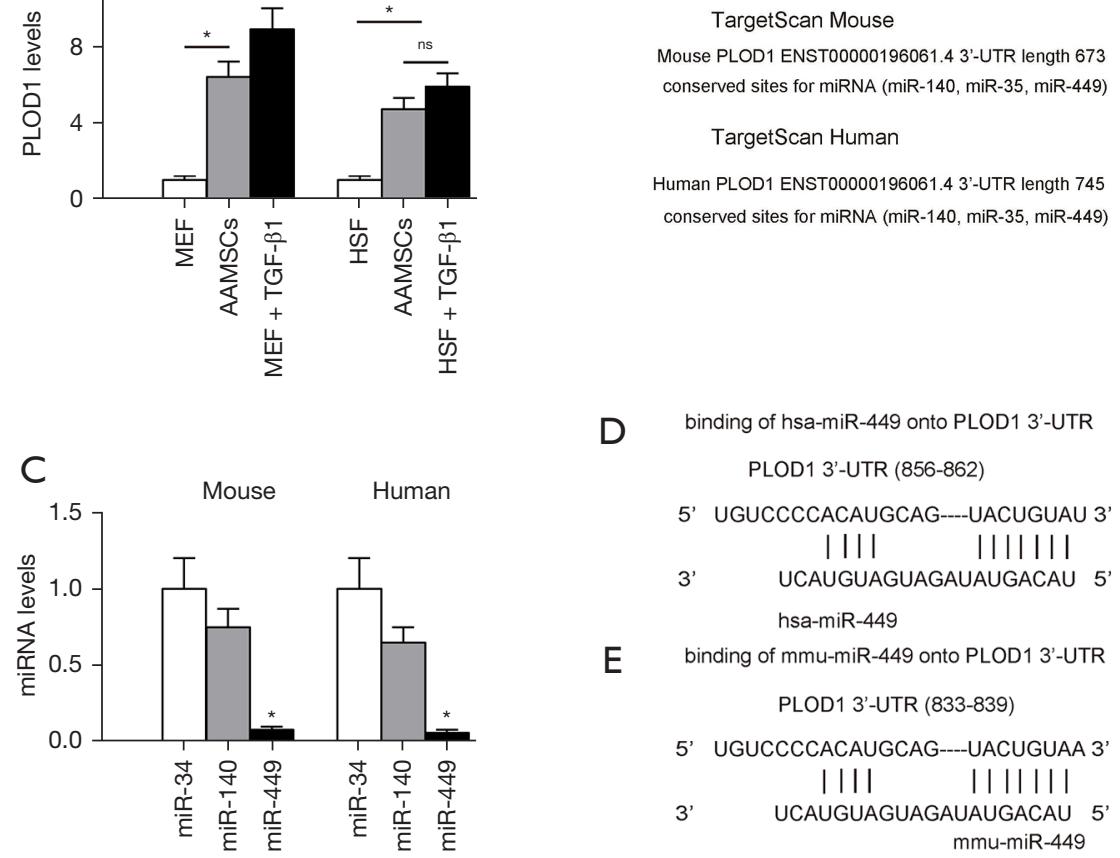

Figure 1 PLOD1-targetting miR-449 is low expressed in AMSCs. (A) PLOD1 protein levels were examined in mouse and human AMSCs by ELISA. MEFs and HSFs treated with/without TGF- $\beta 1$ were used as controls. (B) Bioinformatics tools were used to find 3 PLOD1targeting miRNAs conserved in mouse and human. (C) RT-qPCR for the 3 PLOD1-targeting miRNAs in mouse and human AMSCs. (D,E) The binding sites of miR-449 on human (D) and mouse (E) PLOD1 3'-UTR are shown. *, $\mathrm{P}<0.05$. N=5. ns, non-significant; MEFs, mouse embryonic fibroblasts; HSFs, human skin fibroblasts; AMSCs, adipose-derived mesenchymal stem cells; miRNA, microRNA; RT-qPCR, quantitative reverse transcription polymerase chain reaction; TGF- $\beta 1$, transforming growth factor- $\beta 1$; 3 '-UTR, 3 prime untranslated region.

normalization against internal and experimental controls.

\section{MiRNA target prediction and 3'-UTR luciferase-reporter assay}

The target miRNAs for PLOD1 were selected by TargetScan (http://www.targetscan.org/vert_72/), based on the context++ score (22). The plasmids of the wildtype p3'UTR-PLOD1 and the mutant p3'-UTR-PLOD1-mut were generated. For the dual-luciferase reporter assay, plasmids were co-transfected into AMSCs using Lipofectamine ${ }^{\mathrm{TM}}$ 2000 system (Invitrogen, USA).

\section{Statistical analysis}

GraphPad prism software (version 7, GraphPad Software, Inc., La Jolla, CA, USA) was used in all statistical analyses [one-way analysis of variance (ANOVA) with a Bonferroni correction]. Data were represented as mean \pm standard deviation (SD) and the significance was decided when $\mathrm{P}<0.05$.

\section{Results}

\section{MiR-449 is a PLOD1-targeting miRNA low expressed in} AMSCs

It was shown that PLOD1 has a strong pro-fibrotic effect on fibrosis (5). First, we examined the PLOD1 levels in AMSCs. The levels of PLOD1 in mouse and human AMSCs were thus determined, compared to MEFs and HSFs treated with/without TGF- $\beta 1$, correspondingly. Higher levels of PLOD1 in both mouse and human AMSCs were detected (Figure 1A). Thus, manipulation of PLOD1 levels in AMSCs may enhance their anti-scar potential. Next, we assessed the possibility of inhibiting PLOD1 in AMSCs through miRNAs. Then, we examined the expression levels of 3 PLOD1-targeting miRNAs (miR-34, miR-140, and 
A

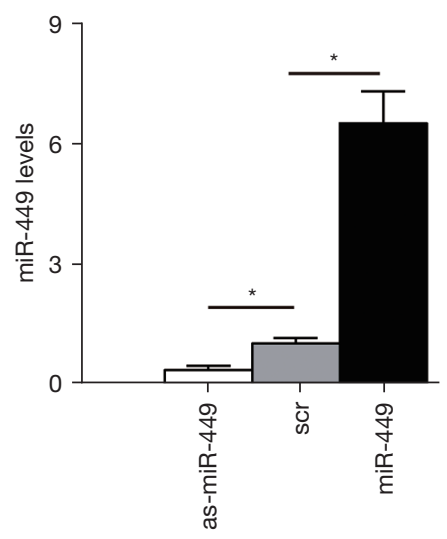

C

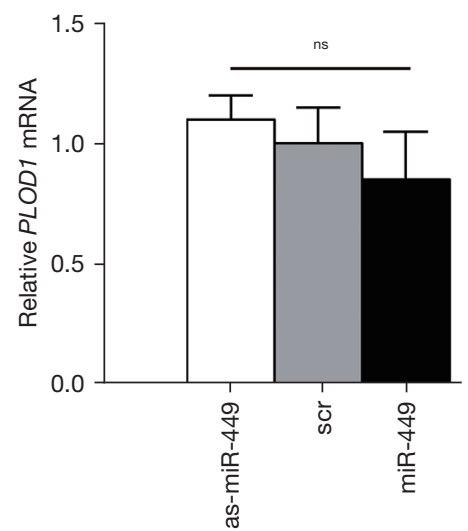

B

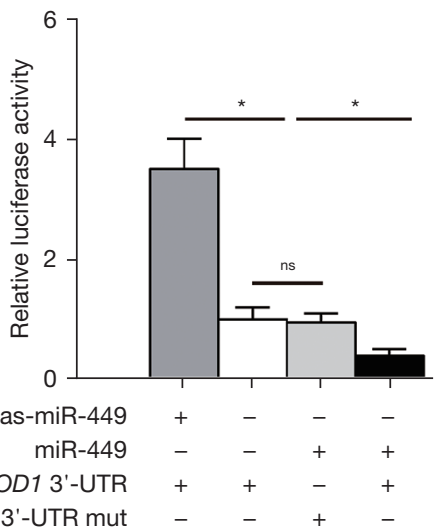

D

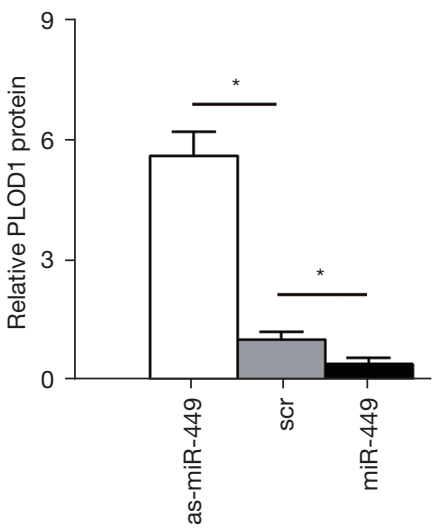

Figure 2 MiR-449 targets 3'-UTR of PLOD1 mRNA to inhibit its protein translation in AMSCs. (A) RT-qPCR for miR-449 in mouse AMSCs cells transfected with plasmids carrying miR-449 or as-miR-449 or scr as a control. (B) The wildtype 3'-UTR of PLOD1 mRNA (wt PLOD1 3'-UTR) and the mutant 3'-UTR of PLOD1 mRNA (the mutant site at the miR-449-binding region) were packaged into the luciferase reporter plasmids to be used in the dual-luciferase reporter assay. (C) RT-qPCR for PLOD1 mRNA in miR-449-modified mouse AMSCs. (D) ELISA for PLOD1 protein in miR-449-modified mouse AMSCs. *, $\mathrm{P}<0.05$. N=5. ns, non-significant; AMSCs, adipose-derived mesenchymal stem cells; miRNA, microRNA; RT-qPCR, quantitative reverse transcription polymerase chain reaction; scr, scrambled sequence; 3'-UTR, 3 prime untranslated region; mRNA, messenger RNA.

miR-449) in mouse and human AMSCs (Figure 1B), and we found that the levels of miR-449 were significantly lower than the other 2 (Figure 1C). The binding sites of miR-449 on human (Figure 1D) and mouse (Figure 1E) PLOD1 3'UTR are shown.

\section{MiR-449 targets 3'-UTR of PLOD1 messenger RNA to inbibit its protein translation in AMSCs}

Next, we examined whether protein translation of PLOD1 may be altered by the bindings of miR-449 to 3'-UTR of PLOD1 messenger RNA (mRNA). First, mouse AMSCs cells (Figure S1) were transfected with plasmids carrying either miR-449, as-miR-449, or control scr. The changes in miR-449 levels were determined by RT-qPCR to assure the effectiveness of the individual plasmids (Figure 2A). Then, the wildtype 3'-UTR of PLOD1 mRNA (wt PLOD1 3'UTR) and the mutant 3'-UTR of PLOD1 mRNA (mut PLOD1 3'-UTR; the mutant site at the miR-449-binding region) were packaged into the luciferase reporter plasmids to be used in the dual-luciferase reporter assay. We found that depletion of miR-449 increased the luciferase activity of wt PLOD1 3'-UTR, while overexpression of miR449 reduced luciferase activity of wt PLOD1 3'-UTR 
but had no effects on mut PLOD1 3'-UTR (Figure 2B). Moreover, alteration of miR-449 levels in mouse AMSCs did not change the mRNA levels of PLOD1 (Figure 2C). However, upregulation of miR-449 significantly decreased PLOD1 protein, while downregulation of miR-449 significantly increased PLOD1 protein in mouse AMSCs (Figure 2D). These results suggest that miR-449 targets 3'UTR of PLOD1 mRNA, resulting in inhibition of protein translation in AMSCs.

\section{Preparation of AMSCs-miR-449 and AMSCs-shPLOD1}

Since we detected high levels of PLOD1 in AMSCs, we planned to increase the anti-scar potential of AMSCs during wound healing through suppression of PLOD1. We used 2 approaches for this. First, AMSCs were transduced with an AAV carrying short-hairpin small interfering RNA for PLOD1 (shPLOD1). Second, AMSCs were transduced with an AAV carrying miR-449. Both viruses also contained a GFP reporter. The AMSCs were transduced with an AAV carrying GFP and a scr was used as a control (Figure $3 A$ ), and the $\mathrm{GFP}^{+}$transduced cells were subsequently purified by flow cytometry (Figure 3B). The levels of PLOD1 were determined in these modified AMSCs by RT-qPCR (Figure $3 C$ ) and ELISA (Figure 3D). We found that expression of miR-449 in AMSCs did not alter PLOD1 mRNA (Figure 3C), but significantly decreased PLOD1 protein (Figure 3D). On the other hand, suppression of PLOD1 in AMSCs by shRNA significantly decreased both PLOD1 mRNA (Figure 3C) and PLOD1 protein (Figure 3D). Moreover, no difference was detected between the PLOD1 protein in AMSCs by miR-449 and by shPLOD1 (Figure 3D). Hence, the 2 approaches may similarly alter PLOD1 protein levels. The AMSCsmiR-449, AMSCs-shPLOD1, and control AMSCs-scr were then applied in the differentiation assays to confirm the maintenance of MSC-phenotype of the transduced cells (Figure 3E-3G).

\section{Transplantation of either AMSCs-miR-449 or AMSCs- shPLOD1 reduces the fibrosis after skin injury in mice}

Then, the in vivo effects by these modifications on fibrosis after skin injury were assessed in mice. The mice were assigned to 3 groups: Group 1: mice received injury and transplantation of control AMSCs-scr; Group 2: mice received injury and transplantation of AMSCs-miR-449; Group 3: mice received injury and transplantation of AMSCs-shPLOD1. We evaluated the effects of transplantation of AMSCs-miR-449, AMSCs-shPLOD1, and control AMSCs-scr on the fibrosis 2 weeks after the skin injury. We found that transplantation of either AMSCsmiR-449 or AMSCs-shPLOD1 significantly reduced the fibrosis at the site of injury, compared to transplantation of AMSCs-scr, shown by representative images (Figure $4 A$ ), and quantification (Figure $4 B$ ). These data suggest that transplantation of either AMSCs-miR-449 or AMSCsshPLOD1 may improve the anti-scar effects of MSCs.

\section{Mechanisms underlying the improved anti-scar potential by PLOD1 suppression in AMSCs}

Finally, we explored the underlying mechanisms. Since macrophages are known key players in fibrosis, we first assessed macrophage number and polarization in these conditions. We did not detect changes in macrophage number (Figure 5A,5B), but found that either AMSCsmiR-449 or AMSCs-shPLOD1 significantly altered macrophage polarization from $\mathrm{M} 2$ to $\mathrm{M} 1$, as evidenced by CD163 positivity (A M2-macrophage marker, Figure 5C,5D). As ROS are critical for serious scar formation, we assessed ROS levels at the site of injury. The results of ELISA (Figure 6A) and DHE assay (Figure 6B) showed that transplantation of either AMSCs-miR-449 or AMSCs-shPLOD1 significantly reduced the ROS levels at the site of the skin injury, compared to transplantation of AMSCs-scr. Transition of myofibroblasts from fibroblasts plays a pivotal role in scar formation. Both $\alpha$-SMA and TGF- $\beta 1$ are specific markers for myofibroblasts and were thus analyzed together with fibrotic markers collagen I and fibronectin at the site of skin injury. We found that transplantation of either AMSCs-miR-449 or AMSCsshPLOD1 significantly reduced the TGF- $\beta 1$ levels (Figure 6C), collagen I levels (Figure 6D), $\alpha$-SMA levels (Figure $6 E$ ), and fibronectin levels (Figure $6 F$ ) at the site of the skin injury, compared to transplantation of AMSCsscr. Thus, suppression of PLOD1 levels in AMSCs either directly by shPLOD1 or indirectly by miR-449 may substantially improve the anti-fibrotic potential of AMSCs during wound healing.

\section{Discussion}

The cellular and molecular mechanisms underlying skin wound repair as well as its failure to heal properly to cause scar formation remains poorly understood. Inferior skin 

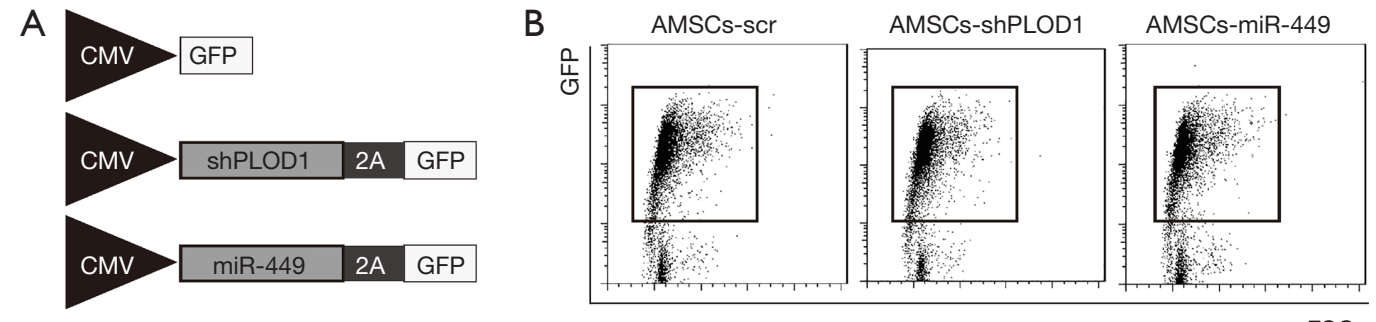

C
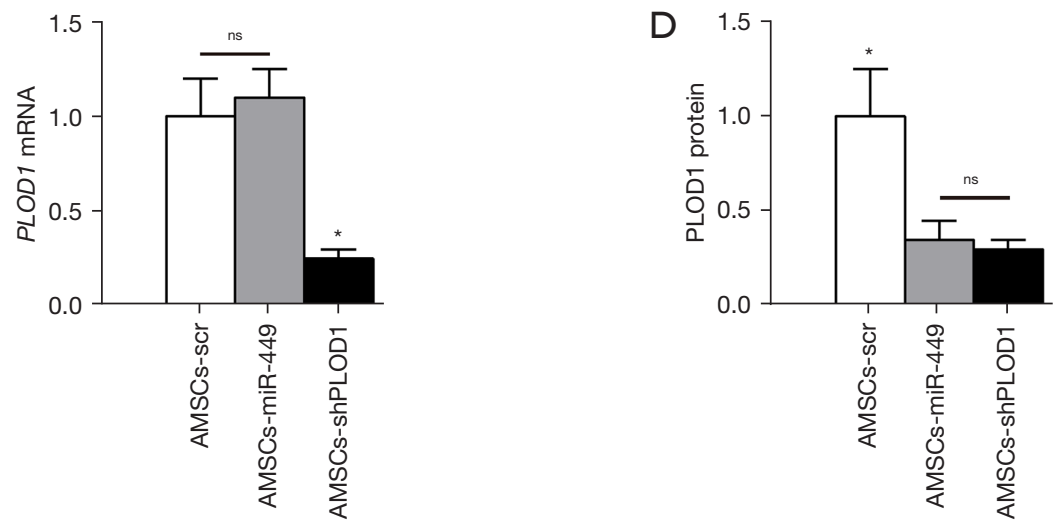

$\mathrm{E}$
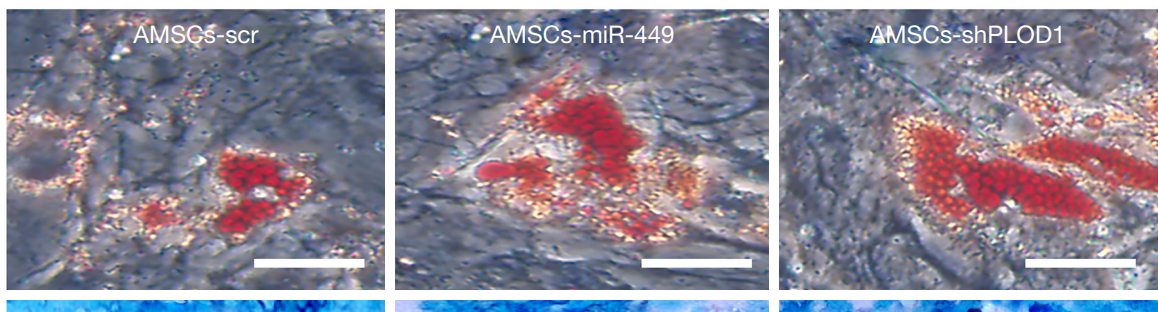

$\mathrm{F}$
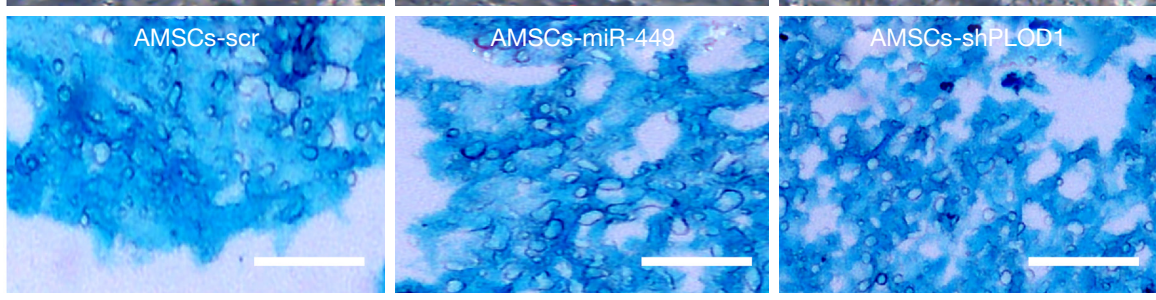

G
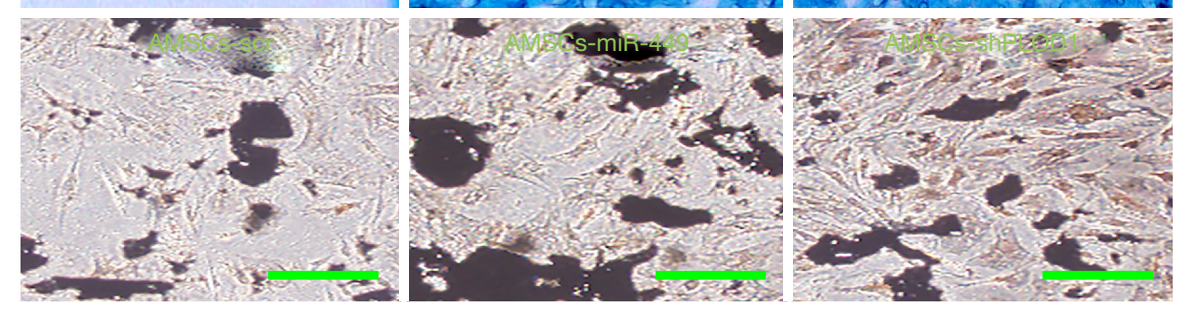

Figure 3 Preparation of AMSCs-miR-449 and AMSCs-shPLOD1. (A) Schematic of AAVs: [1] an AAV carrying GFP and scr; [2] an AAV carrying short-hairpin small interfering RNA for PLOD1 (shPLOD1) and a GFP reporter under control of a CMV promoter; [3] an AAV carrying miR-449 and a GFP reporter under control of a CMV promoter. (B) Transduced mouse AMSCs were purified based on GFP expression by flow cytometry. (C) RT-qPCR for PLOD1 levels in AMSCs-miR-449, AMSCs-shPLOD1 and control AMSCs-scr. (D) ELISA for PLOD1 levels in AMSCs-miR-449, AMSCs-shPLOD1 and control AMSCs-scr. (E) Oil red O staining to evaluate adipogenic induction (scale bars are $50 \mu \mathrm{m}$ ). (F) Alcian blue staining to evaluate chondrogenic induction (scale bars are $50 \mu \mathrm{m}$ ). (G) Von Kossa staining to evaluate osteogenic induction (scale bars are $50 \mu \mathrm{m}$ ). *, $\mathrm{P}<0.05$. N=5. ns, not significant; PLOD1, procollagen-lysine 1,2-oxoglutarate 5-dioxygenase 1; AAV, adeno-associated virus; CMV, cytomegalovirus; AMSCs, adipose-derived mesenchymal stem cells; miRNA, microRNA; RT-qPCR, quantitative reverse transcription polymerase chain reaction; scr, scrambled sequence; ELISA, enzyme-linked immunosorbent assay. 


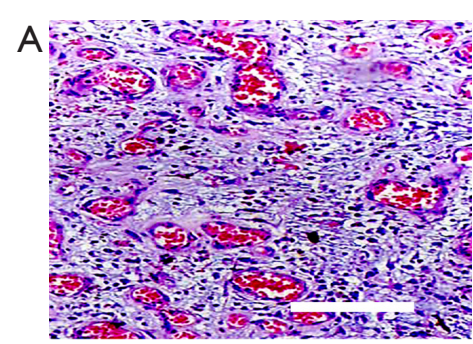

AMSCs-scr

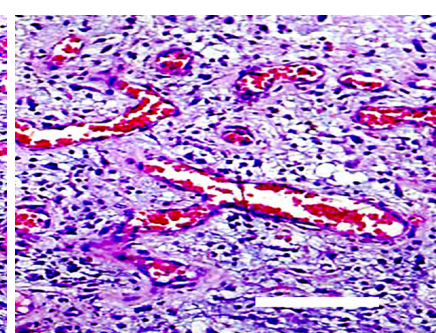

AMSCs-miR-449

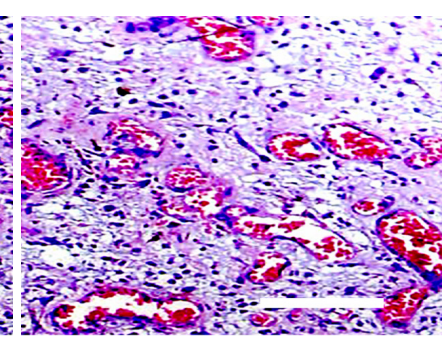

AMSCs-shPLOD1

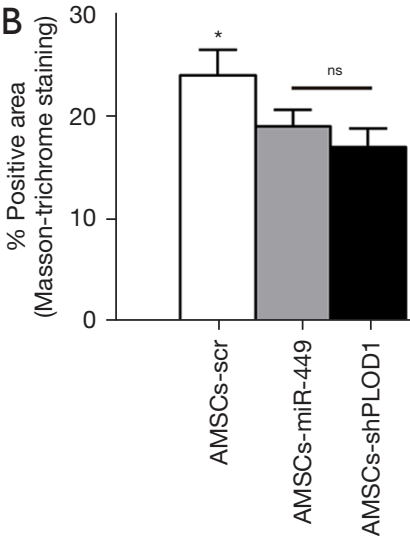

Figure 4 Transplantation of either AMSCs-miR-449 or AMSCs-shPLOD1 decreases the fibrosis after skin injury in mice. (A,B) The effects of transplantation of AMSCs-miR-449, AMSCs-shPLOD1 and control AMSCs-scr on the fibrosis were evaluated by Masson-trichrome staining 2 weeks after the skin injury in mice, shown by representative images (A), and quantification (B). * $\mathrm{P}<0.05$. $\mathrm{N}=10$. Scale bars are 50 $\mu \mathrm{m}$. ns, not significant; AMSCs, adipose-derived mesenchymal stem cells; PLOD1, procollagen-lysine 1,2-oxoglutarate 5-dioxygenase 1; scr, scrambled sequence.

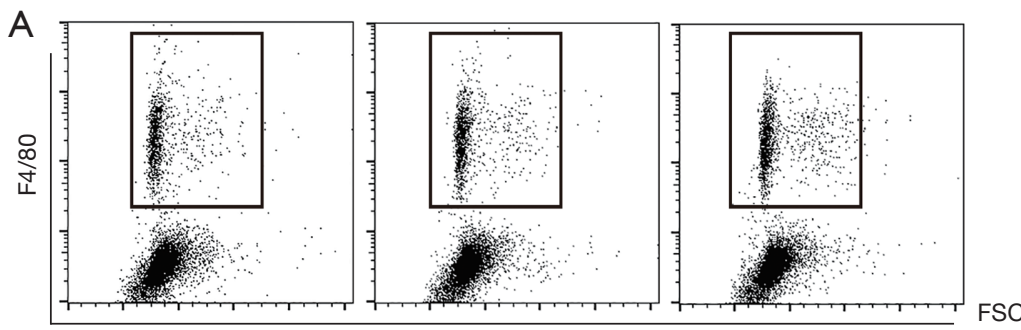

B
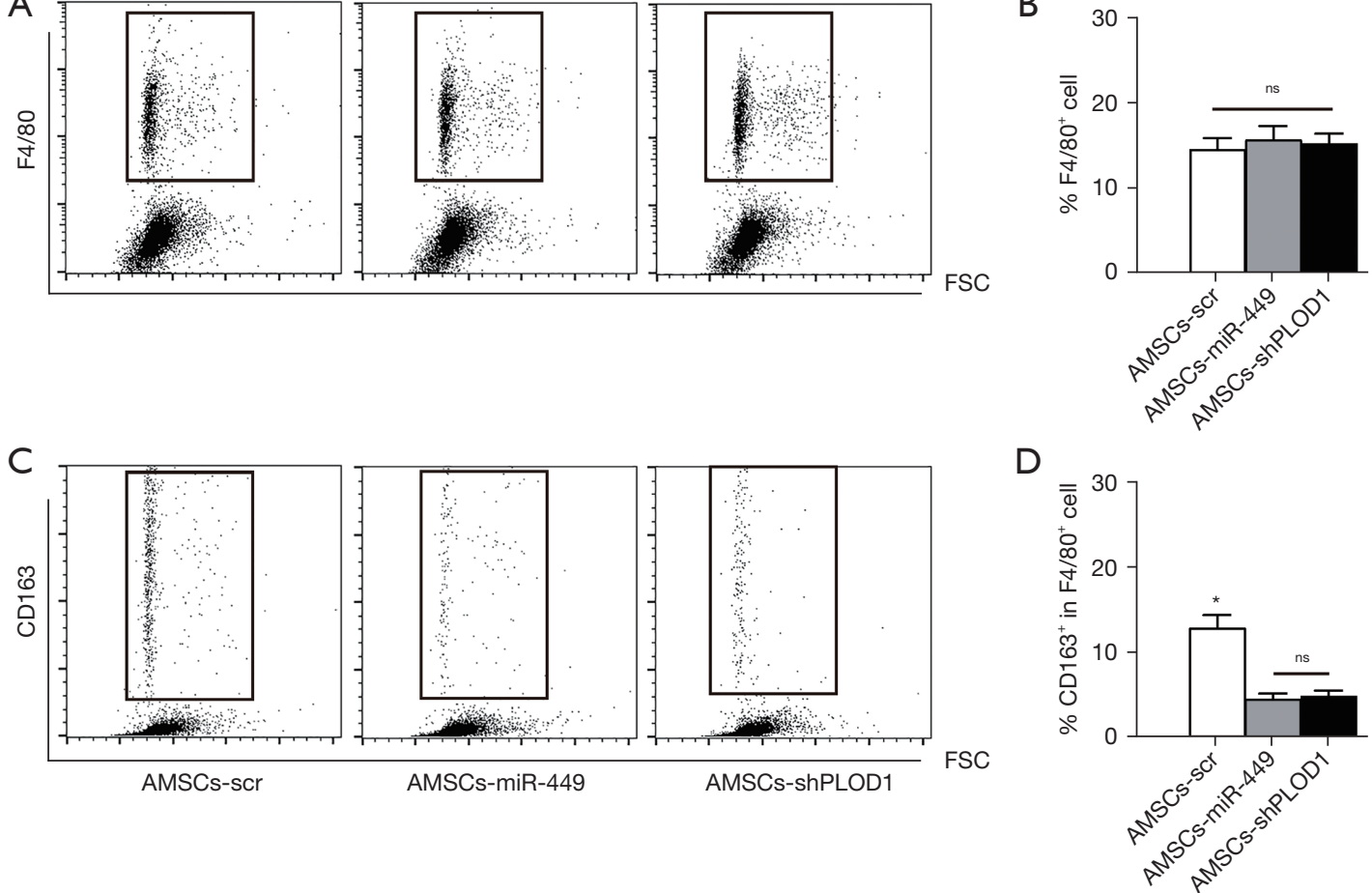

Figure 5 Effects on macrophage polarization. (A,B) The effects of transplantation of AMSCs-miR-449, AMSCs-shPLOD1 and control AMSCs-scr on the macrophage number by flow cytometry on F4/80, shown by representative flow charts (A) and by quantification (B). (C,D) The effects of transplantation of AMSCs-miR-449, AMSCs-shPLOD1 and control AMSCs-scr on the macrophage polarization by flow cytometry on CD163 in F4/80 cells, shown by representative flow charts (C) and by quantification (D). * $\mathrm{P}<0.05$. N=10. ns, not significant; AMSCs, adipose-derived mesenchymal stem cells; PLOD1, procollagen-lysine 1,2-oxoglutarate 5-dioxygenase 1; scr, scrambled sequence. 
A

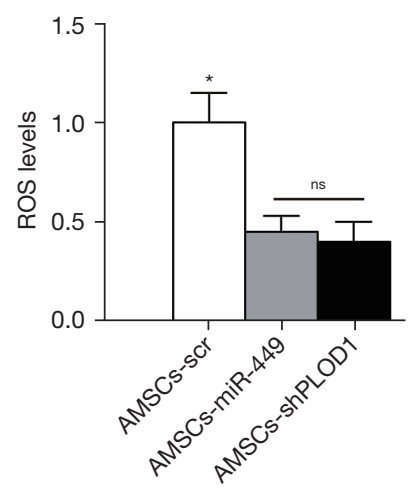

C

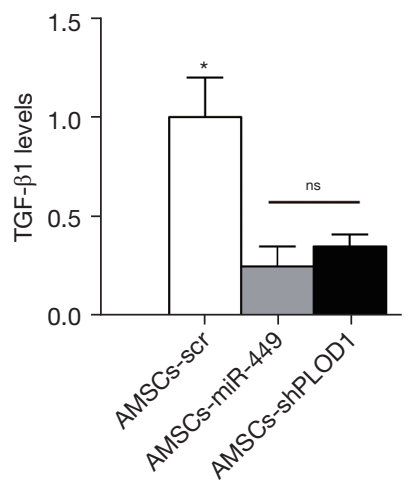

E

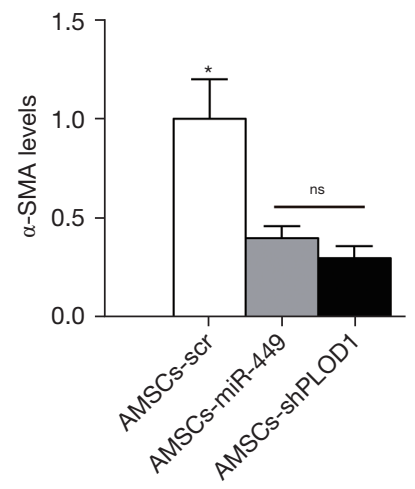

B
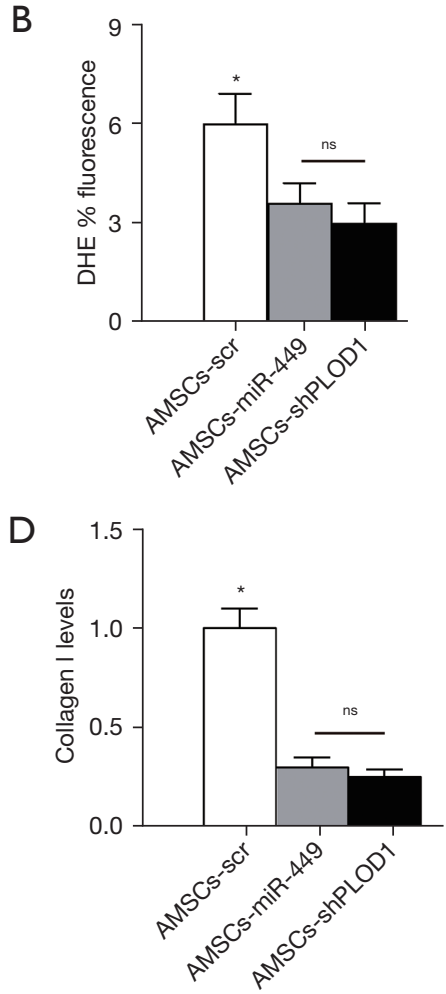

$\mathrm{F}$

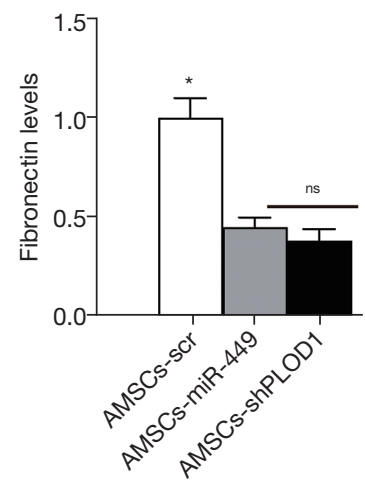

Figure 6 Mechanisms underlying the improved anti-scar potential by PLOD1 suppression in AMSCs. (A) ELISA for ROS; (B) DHE assay; (C-F) ELISA for TGF- $\beta 1$ (C), collagen I (D), $\alpha$-SMA (E) and fibronectin (F), at the site of the skin injury. *, P<0.05. N=10. ns, not significant; $\alpha$-SMA, $\alpha$-smooth muscle actin; DHE, dihydroethidium; ELISA, enzyme-linked immunosorbent assay; ROS, reactive oxygen species; PLOD1, procollagen-lysine 1,2-oxoglutarate 5-dioxygenase 1; scr, scrambled sequence; TGF- $\beta 1$, transforming growth factor $\beta 1$.

wound healing affects millions of people worldwide each year (9-11). A common denominator of fibrosis and scar formation is the presence of elevated levels of collagen type I, which is deposited by myofibroblasts activated by profibrotic cytokines like TGF- $\beta 1$. Pyridinoline crosslinks are then required to stabilize collagen fibrils, and this process is regulated by PLOD family members including PLOD1, PLOD2, and PLOD3. Collagen containing pyridinoline cross-links are resistant to collagenasemediated degradation and thereby cause fibrotic lesion. Thus, reduction in pyridinoline cross-links may enable the formation of a non-fibrotic matrix to attenuate scar formation (5). Here, we detected high PLOD1 in AMSCs and thus reduction in PLOD1 levels appeared to be an attractive strategy to interfere with a critical step during fibrosis and scar formation. 
In the current study, we used 2 strategies to suppress PLOD1 levels in AMSCs, and both achieved satisfactory effects on the anti-scar potential of AMSCs. We feel that either strategy could have its advantage and disadvantage. For direct suppression of PLOD1 by shPLOD1, the advantage is that the depletion of PLOD1 is straightforward and unlikely has any off-targeting effects as a scr was used as a control. However, both PLOD1 mRNA and protein were altered by shPLOD1, and thus the effects of reduced PLOD1 mRNA on the cell biology remains unknown. For indirect suppression of PLOD1 by miR-449, the advantage is that the PLOD1 mRNA is not altered and thus there should be little feedback effects at the mRNA level. Moreover, alteration of a protein level through posttranscriptional control is always an effective and healthy way to modulate cells. However, miR-449 may have target genes other than PLOD1. Thus, the non-specific effects on the AMSCs as well as their therapeutic effects on wound healing are still uncertain and require further investigation. In future, further analyses of the non-PLOD1 targets for miR-449 may be helpful for the effects of miR-449modulation on the therapeutic effects of miR-449.

The effects of altering PLOD1 levels in AMSCs on macrophage polarization have been suggested in cancer studies (23) and here we showed that it may be also important in regulation of fibrosis. The inhibition of M2like fibrotic macrophages here and the inhibition of M2-like tumor-associated macrophages in cancer by PLOD1 were similar.

To summarize, here we have provided evidence to demonstrate a useful approach by altering AMSCs to improve their application in attenuating scar formation after skin injury.

\section{Acknowledgments}

Funding: This work was supported by internal funding from Shanghai Jiao Tong University.

\section{Footnote}

Reporting Checklist: The authors have completed the ARRIVE reporting checklist. Available at https://dx.doi. org/10.21037/atm-21-4978

Data Sharing Statement: Available at https://dx.doi. org/10.21037/atm-21-4978

Conflicts of Interest: All authors have completed the ICMJE uniform disclosure form (available at https://dx.doi. org/10.21037/atm-21-4978). The authors have no conflicts of interest to declare.

Ethical Statement: The authors are accountable for all aspects of the work in ensuring that questions related to the accuracy or integrity of any part of the work are appropriately investigated and resolved. The present study was approved by the Research and Animal Ethics Association of Shanghai Jiao Tong University (No. XHEC-F-2021-063), in accordance with the guidance from Shanghai Jiao Tong University for the Care and Use of Laboratory Animals.

Open Access Statement: This is an Open Access article distributed in accordance with the Creative Commons Attribution-NonCommercial-NoDerivs 4.0 International License (CC BY-NC-ND 4.0), which permits the noncommercial replication and distribution of the article with the strict proviso that no changes or edits are made and the original work is properly cited (including links to both the formal publication through the relevant DOI and the license). See: https://creativecommons.org/licenses/by-nc-nd/4.0/.

\section{References}

1. Visscher MO, Bailey JK, Hom DB. Scar treatment variations by skin type. Facial Plast Surg Clin North Am 2014;22:453-62.

2. Li X, Li J, Ju X, et al. Abdominal scar characteristics as a predictor of cervical stenosis after abdominal radical trachelectomy. Oncotarget 2016;7:37755-61.

3. Rhett JM, Ghatnekar GS, Palatinus JA, et al. Novel therapies for scar reduction and regenerative healing of skin wounds. Trends Biotechnol 2008;26:173-80.

4. Beanes SR, Dang C, Soo C, et al. Skin repair and scar formation: the central role of TGF-beta. Expert Rev Mol Med 2003;5:1-22.

5. Anum EA, Hill LD, Pandya A, et al. Connective tissue and related disorders and preterm birth: clues to genes contributing to prematurity. Placenta 2009;30:207-15.

6. Perciani CT, MacParland SA. Lifting the veil on macrophage diversity in tissue regeneration and fibrosis. Sci Immunol 2019;4:eaaz0749.

7. Li Q, Zhang C, Fu X. Will stem cells bring hope to pathological skin scar treatment? Cytotherapy 2016;18:943-56.

8. Jackson WM, Nesti LJ, Tuan RS. Mesenchymal stem cell 
therapy for attenuation of scar formation during wound healing. Stem Cell Res Ther 2012;3:20.

9. Wang Y, Chu Y, Yue B, et al. Adipose-derived mesenchymal stem cells promote osteosarcoma proliferation and metastasis by activating the STAT3 pathway. Oncotarget 2017;8:23803-16.

10. Chen KH, Chen CH, Wallace CG, et al. Intravenous administration of xenogenic adipose-derived mesenchymal stem cells (ADMSC) and ADMSC-derived exosomes markedly reduced brain infarct volume and preserved neurological function in rat after acute ischemic stroke. Oncotarget 2016;7:74537-56.

11. Zhong Z, Gu H, Peng J, et al. GDNF secreted from adipose-derived stem cells stimulates VEGF-independent angiogenesis. Oncotarget 2016;7:36829-41.

12. van den Broek LJ, Niessen FB, Scheper RJ, et al. Development, validation and testing of a human tissue engineered hypertrophic scar model. ALTEX 2012;29:389-402.

13. Hiwatashi N, Hirano S, Suzuki R, et al. Comparison of ASCs and BMSCs combined with atelocollagen for vocal fold scar regeneration. Laryngoscope 2016;126:1143-50.

14. Valerie A, Vassiliki K, Irini M, et al. Adipose-Derived Mesenchymal Stem Cells in the Regeneration of Vocal Folds: A Study on a Chronic Vocal Fold Scar. Stem Cells Int 2016;2016:9010279.

15. van den Bogaerdt AJ, van der Veen VC, van Zuijlen PP, et al. Collagen cross-linking by adipose-derived mesenchymal stromal cells and scar-derived mesenchymal cells: Are mesenchymal stromal cells involved in scar formation? Wound Repair Regen 2009;17:548-58.

Cite this article as: $\mathrm{Xu} \mathrm{M,} \mathrm{Fang} \mathrm{S,} \mathrm{Xie} \mathrm{A.} \mathrm{Posttranscriptional}$ control of PLOD1 in adipose-derived stem cells regulates scar formation through altering macrophage polarization. Ann Transl Med 2021;9(20):1573. doi: 10.21037/atm-21-4978
16. Seok H, Ham J, Jang ES, et al. MicroRNA Target Recognition: Insights from Transcriptome-Wide NonCanonical Interactions. Mol Cells 2016;39:375-81.

17. Marcet B, Chevalier B, Luxardi G, et al. Control of vertebrate multiciliogenesis by miR-449 through direct repression of the Delta/Notch pathway. Nat Cell Biol 2011;13:693-9.

18. Feng $M$, Yu Q. miR-449 regulates CDK-Rb-E2F1 through an auto-regulatory feedback circuit. Cell Cycle 2010;9:213-4.

19. Li Z, Huang X, Xu J, et al. miR-449 overexpression inhibits papillary thyroid carcinoma cell growth by targeting RET kinase- $\beta$-catenin signaling pathway. Int J Oncol 2016;49:1629-37.

20. Guo J, Hu H, Gorecka J, et al. Adipose-derived mesenchymal stem cells accelerate diabetic wound healing in a similar fashion as bone marrow-derived cells. Am J Physiol Cell Physiol 2018;315:C885-96.

21. Hu M, Wu Y, Yang C, et al. Novel Long Noncoding RNA lnc-URIDS Delays Diabetic Wound Healing by Targeting Plod1. Diabetes 2020;69:2144-56.

22. Agarwal V, Bell GW, Nam JW, et al. Predicting effective microRNA target sites in mammalian mRNAs. Elife 2015;4:e05005.

23. Wang Z, Shi Y, Ying C, et al. Hypoxia-induced PLOD1 overexpression contributes to the malignant phenotype of glioblastoma via NF- $\kappa \mathrm{B}$ signaling. Oncogene 2021;40:1458-75.

(English Language Editor: J. Jones) 


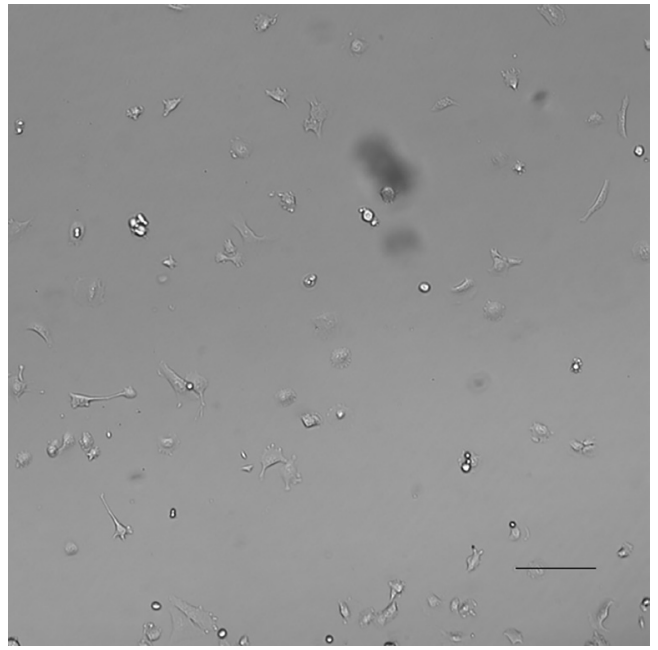

Figure S1 Representative brightfield images for AMSCs in culture. The scale bar is $50 \mu \mathrm{m}$. 